\title{
Rhizosphere Fungal Community Dynamics Associated with Rehmannia glutinosa Replant Disease in a Consecutive Monoculture Regime
}

\author{
Linkun Wu, Jun Chen, Muhammad Umar Khan, Juanying Wang, Hongmiao Wu, Zhigang Xiao, \\ Zhongyi Zhang, and Wenxiong Lin ${ }^{\dagger}$
}

First, second, third, fourth, fifth, sixth, and eighth authors: College of Life Sciences and Fujian Provincial Key Laboratory of Agroecological Processing and Safety Monitoring; seventh and eighth authors: Key Laboratory of Crop Ecology and Molecular Physiology, Fujian Province University; and seventh author: College of Crop Science, Fujian Agriculture and Forestry University, Fuzhou 350002, Fujian, P.R. China. Accepted for publication 2 July 2018.

\begin{abstract}
Consecutive monoculture of Rehmannia glutinosa in the same field leads to a severe decline in both quality and yield of tuberous roots, the most useful part in traditional Chinese medicine. Fungi are an important and diverse group of microorganisms in the soil ecosystem and play crucial roles in soil health. In this study, high-throughput pyrosequencing of internal transcribed spacer 2 ribosomal DNA amplicons was applied to gain insight into how consecutive monoculture practice influence and stimulate $R$. glutinosa rhizosphere and bulk soil fungal communities. The results from nonmetric multidimensional scaling ordination and clustering analysis revealed distinctive differences between rhizosphere and bulk soil fungal communities. However, longer-term monocultured bulk soils were more similar to the rhizosphere soils in comparison with the shorterterm monocultured bulk soils. Moreover, consecutive monoculture caused a gradual shift in the composition and structure of the soil fungal community. The cultivation of this plant led to the appearance of some exclusive operational taxonomic units in rhizosphere or bulk soils that were assigned to the genera Fusarium, Rhizoctonia, and so on. Furthermore, the sum of

the relative abundance of species of Fusarium, Cylindrocarpon, and Gibberella (belonging to the family Nectriaceae); Rhizoctonia, Thanatephorus, and Ceratobasidium (belonging to the family Ceratobasidiaceae); and Lectera and Plectosporium (belonging to the family Plectosphaerellaceae) was significantly higher in consecutively monocultured (CM) than in newly planted (NP) soil in both rhizosphere and bulk soils. In particular, Fusarium abundance was significantly higher in CM than in NP in the rhizosphere, and higher in rhizosphere soils than in bulk soils for each treatment. A pathogenicity test showed that both Fusarium strains isolated were pathogenic to $R$. glutinosa seedlings. In addition, the culture filtrate and mycotoxins produced by Fusarium oxysporum significantly repressed the growth of the antagonistic bacterium, Pseudomonas aeruginosa. In conclusion, consecutive monoculture of $R$. glutinosa restructured the fungal communities in both rhizosphere and bulk soils but bulk effects developed more slowly over time in comparison with rhizosphere effects. Furthermore, microbial interactions might lead to a reduction in the abundance of beneficial microbes.
\end{abstract}

Rehmannia glutinosa, a perennial herbaceous plant, belongs to the family Scrophulariaceae. The tuberous root of this plant is widely used in traditional Chinese medicine because it contains various pharmaceutically active compounds, including rehmannin, amino acids, vitamins, iridoids, aucubin, and sugars ( $\mathrm{Li}$ et al. 2017). These active components exhibit a wide range of pharmacological actions related to the blood, immune, endocrine, cardiovascular, and nervous systems. R. glutinosa has become one of the 50 fundamental herbs used in traditional Chinese medicine (Park et al. 2009). Unfortunately, both yield and quality significantly decline when $R$. glutinosa is consecutively monocultured in the same field (Wu et al. 2015). This decline is commonly described as "consecutive monoculture problems" or "replant disease" (Yang et al. 2014). Consecutive monoculture of this plant in the same field

${ }^{\dagger}$ Corresponding author: Wenxiong Lin; E-mail: lwx@fafu.edu.cn

Funding: This work was supported by the Natural Science Foundation of Fujian Province (grant number 2017J01803), the Outstanding Youth Scientific Fund of Fujian Agriculture and Forestry University (grant number XJQ201501), the Program for Innovative Research Team in Fujian Agricultural and Forestry University (grant numbers 712018009 and CXZX2017302), the National Natural Science Foundation of China (grant numbers 81573530 and 81303170), the National Key Research and Development Program of China (grant number 2017YFC1700705), and Fujian-Taiwan Joint Innovative Center for Germplasm Resources and Cultivation of Crop (FJ 2011 program number 2015-75).

*The $\boldsymbol{e}$-Xtra logo stands for "electronic extra" and indicates that six supplementary figures and three supplementary tables are published online.

(C) 2018 The American Phytopathological Society results in a total crop failure of underground tubers (Supplementary Fig. S1). Newly planted (NP) plants (grown in a paddock where $R$. glutinos $a$ had not been planted for more than 10 years) exhibited normally expanded tuberous roots and consecutively monocultured (CM) plants exhibited poor growth, adventitious fibrous roots, and serious root rot disease. Fields used for $R$. glutinosa production are typically replanted every 15 to 20 years (Yang et al. 2011). Additionally, to obtain the highest-quality medicinal materials, also known as geo-authentic herbs, they should be produced in their geoauthentic areas (indigenous regions) (Han et al. 2016). That is why high-quality tuberous roots of $R$. glutinosa are mostly produced in Jiaozuo City, Henan Province, China ( $\left.34^{\circ} 56^{\prime} \mathrm{N}, 112^{\circ} 58^{\prime} \mathrm{E}\right)$, known as the geo-authentic area of this plant. $R$. glutinosa planted in fields outside of the geo-authentic production zone cannot be assured of good quality (Wu et al. 2015). Therefore, it has become urgent to explore the formation mechanisms of replant disease that occurs in $R$. glutinosa cultivation.

Previous studies of replant disease existing in different crops focused on soil physicochemical properties and the autotoxicity of root exudates (Chen et al. 2015; Huang et al. 2013). However, many studies also indicated that replant disease was not directly related to the deficiency of soil nutrients and direct allelopathy of root exudates (Leinfelder and Merwin 2006; H. M. Wu et al. 2016). Similar examples could also be found in R. glutinosa (Wu et al. 2013, 2015). The rhizosphere, a zone of soil immediately surrounding the roots, is active and dynamic; there, newly generated carbon and ancient carbon are rich and available for microbial growth (Haichar et al. 2008). By contrast, available organic carbon sources in bulk soil are relatively lower. However, the bulk soil 
network was referred to as the "seed bank" from which rhizosphere networks are recruited (de Vries and Wallenstein 2017). Moreover, root exudates released into the rhizosphere could select certain microorganisms from the bulk soil (de Vries and Wallenstein 2017; Haichar et al. 2008; Miethling et al. 2000). The collective genome of the root-associated microbiome is referred to as the plant's second genome, which is critical for plant growth, health, and fitness (Berendsen et al. 2012). Now, the propensity toward recognition of the ecological linkages between aboveground and belowground biota is increasing (Lakshmanan et al. 2014; Wardle et al. 2004). Current research into replant disease has mainly focused on the effects of the soil microbiome on soil health and plant growth (Hewavitharana and Mazzola 2016; Jiang et al. 2017; Mazzola et al. 2002). For example, peach tree replanting could selectively influence the microbial community structure with an increase in phytotoxic microorganisms (Benizri et al. 2005). Long-term monoculture of banana significantly altered the bacterial and fungal community structures, leading to the depletion of indigenous microbes and an outbreak of pathogens (Shen et al. 2018). The number and diversity indices of rhizospheric bacteria decreased with the increasing years of consecutive monoculture in Panax notoginseng (Tan et al. 2017). It is now widely recognized that replant disease is likely to be driven by the successional changes in root-associated microbial community induced by root exudates (Li et al. 2014a; Zhou and Wu 2012).

Fungi, an important and diverse group of microorganisms in the soil ecosystem, play crucial roles in soil quality and crop health because of their multiple functions, including positive and negative associations (Manici and Caputo 2009; Xu et al. 2012). Li et al. (2014b) attributed the problems associated with consecutive monoculture in peanut to the accumulations of fungal pathogen loads at the expense of plant-beneficial fungi in the soil. To date, many fungi species, including Fusarium, Aspergillus, and Phoma spp., were reported to be the main agents of the root rot complex in herbaceous crops and medicinal plants in intensive cropping systems (Li et al. 2014b; Manici and Caputo 2009; Wu et al. 2013; Xiong et al. 2017). Previous studies pointed out that consecutive monoculture of Pseudostellaria heterophylla could significantly increase the relative abundances of species of Fusarium, Trichocladium, Myrothecium, Simplicillium, and so on but decrease the relative abundance of Penicillium spp. (L. K. Wu et al. 2016). Similarly, soil fungal community structure and diversity were significantly influenced by consecutive monoculture of Panax notoginseng, and Myrmecridium, Phaeosphaeria, Fusarium, and Phoma spp. were found to be better adapted to colonization of diseased plants (Dong et al. 2016).

Understanding of the successive changes in microbial community composition and interaction networks in a long-term monoculture regime is necessary to manipulate and manage the soil microbiome to improve soil health and increase crop production. Our previous study demonstrated that $R$. glutinosa monoculture resulted in distinct bacterial community variation, with a reduction in the abundance of beneficial microbes (i.e., Pseudomonas spp.) (Wu et al. 2015, 2018). However, little is known about the secondary metabolites (i.e., mycotoxins) of specific fungal pathogens on the growth of the antagonistic Pseudomonas spp. In addition, although the comparison of the variability in rhizosphere fungal community composition between the NP and 2-year CM R. glutinosa has also been studied (Yang et al. 2016), the responses of soil microbial community under longer-term $R$. glutinosa monoculture and the variations of bulk and rhizosphere fungal communities have not yet been fully elucidated. Therefore, in this study, parallel pyrosequencing of internal transcribed spacer 2 (ITS2) ribosomal DNA (rDNA) amplicons combined with a culture-dependent approach was applied to characterize the bulk and rhizosphere soil fungal communities under 4-year consecutive monoculture of $R$. glutinosa. We hypothesized that the consecutive monoculture of $R$. glutinosa could shape the soil fungal community structure and result in the accumulation of certain fungal pathogens associated with replant disease at the expense of beneficial microbes.

\section{MATERIALS AND METHODS}

Field experiment and soil sampling. Experimental $R$. glutinosa 'Wen 85-5' plants were grown in Jiaozuo City, Henan Province, China $\left(34^{\circ} 56^{\prime} \mathrm{N}, 112^{\circ} 58^{\prime} \mathrm{E}\right)$. This city is well known as the geoauthentic zone for $R$. glutinosa cultivation, with an annual mean temperature of $14.3^{\circ} \mathrm{C}$ and an annual mean precipitation of $552 \mathrm{~mm}$. $R$. glutinosa was generally planted on 15 April and harvested on 30 October of the same year. After harvest, the paddock was kept fallow till replanting on 15 April of the next year. Five cropping patterns of $R$. glutinosa were established, including a control with no $R$. glutinosa cultivation (CK); NP; and 2-year (SM), 3-year (TM), and 4-year (FM) CM plots. NP plants were grown in a paddock where $R$. glutinosa had not been planted for more than 10 years. NP plots were established each year. The CM plants were grown in a paddock where $R$. glutinosa had been grown in the previous year. All plots (NP, SM, TM, and FM) were planted in different years and then sampled at the same time. All five cropping patterns were conducted in a single field site (every year, a section of the field was kept fallow to establish the NP plots in the next year) to ensure uniform growth conditions among treatments. The soil physiochemical parameters for the field site were as follows: sandy loam soil, $\mathrm{pH} 7.52$, soil organic matter $=10.32 \mathrm{~g} \cdot \mathrm{kg}^{-1}$, available nitrogen $=21.34 \mathrm{mg} \cdot \mathrm{kg}^{-1}$, available phosphorus $=47.02 \mathrm{mg} \cdot \mathrm{kg}^{-1}$, and available potassium $=251.34 \mathrm{mg} \cdot \mathrm{kg}^{-1}$. All plots were subjected to the same fertilization and field management during the entire experimental period.

Soil samples were collected from five random locations within each plot and mixed together to make composite samples. For each soil category, there were three biological replicates. Because both rhizosphere and bulk soils of $R$. glutinosa were collected at the root expansion stage (5 months after planting), we obtained nine different soil categories: control soil (CK), NP rhizosphere soil (RNP), SM rhizosphere soil (RSM), TM rhizosphere soil (RTM), FM rhizosphere soil (RFM), NP bulk soil (BNP), SM bulk soil (BSM), TM bulk soil (BTM), and FM bulk soil (BFM). Soil samples from all different treatments were collected at the same time in the same year. For soil sampling, fresh plants were carefully uprooted from the soil with a forked spade and their tuberous roots were shaken to remove loosely attached soil. The soil that tightly attached to tuberous roots was sampled as the rhizosphere soil, while the soil that was 15 to $20 \mathrm{~cm}$ away from roots was sampled as the bulk soil. All collected soil samples were sieved through 2-mm mesh to remove plant roots, leaf debris, and insects for further analyses.

Fungal community analysis by ITS2 pyrosequencing. Total soil DNA was extracted by using a SoilGen DNA kit (CWBIO, Beijing, China), following the manufacturer's instructions. DNA was diluted to $1 \mathrm{ng} / \mu \mathrm{l}$ and then used for ITS2 amplification using primers ITS3-KYO2 (5'-GATGAAGAACGYAGYRAA-3') and ITS4 (5'-TCCTCCGCTTATTGATATGC-3') (Toju et al. 2012). Each soil category had three replicates. Polymerase chain reaction (PCR) products obtained were migrated in agarose gel, extracted, and purified using a Gel Extraction Spin-50 kit (Chromous, Bangalore, India). Purified libraries were sequenced on an Illumina HiSeq2500 platform.

Bioinformatic analysis of pyrosequencing data. The sequence reads were assigned to the individual sample based on their unique barcodes. The low-quality sequences were excluded according to the QIIME (V1.7.0) quality-controlled process. The obtained effective sequences were assigned to operational taxonomic units (OTU) using Mothur (v.1.34.0), with a cutoff of $97 \%$ similarity. Species annotation was carried out via the Ribosomal Database Project Bayesian Classifier (Deshpande et al. 2016).

Singletons that occurred only once in the entire data set were removed before further analysis. Then, the abundances of OTU 
were calculated by normalizing to the total of the reads per sample. The $\alpha$ and $\beta$ diversity analyses were performed based on the normalized data. The $\alpha$ diversity was applied to analyze species complexity for a sample through three indices, including Pielou's evenness index and Shannon and Simpson diversity indices.

The $\beta$ diversity analyses, including nonmetric multidimensional scaling (NMDS) ordination and group-average clustering analysis based on the relative abundances of OTU (logarithmic transformation, standardization, and Bray-Curtis dissimilarity matrix), were used to examine relative similarities of microbial communities. Analysis of similarity (ANOSIM), a nonparametric statistical test, was used to examine the statistical significance between samples based on the relative abundances of the fungal genera (logarithmic transformation, standardization, and Bray-Curtis dissimilarity matrix). Furthermore, similarity percentage (SIMPER) analysis (logarithmic transformation and standardization) was performed to determine the taxa contributing to the dissimilarities between samples. The above multivariate statistical analyses were implemented in the PRIMER V5 software package (PRIMER-E Ltd., Plymouth, UK). Venn diagram analysis of OTU was carried out using R software (version 3.4.1) to detect the exclusive and shared OTU between samples.

Isolation and identification of Fusarium and pathogenicity test. Potato dextrose agar (PDA) was used to isolate potential fungal pathogens from the rhizosphere soil of monocultured $R$. glutinosa plants with root rot symptoms. In detail, $10 \mathrm{~g}$ of the collected fresh soils were put into a flask containing $90 \mathrm{ml}$ of sterile water $\left(10^{-1}\right.$ dilution $)$. After $10 \mathrm{~min}$ of shaking at $180 \mathrm{rpm}$ and $28^{\circ} \mathrm{C}, 200 \mu \mathrm{l}$ of the $10^{-1}$ soil suspension was plated onto PDA medium containing streptomycin at $100 \mu \mathrm{g} \cdot \mathrm{ml}^{-1}$ and ampicillin at $100 \mu \mathrm{g} \cdot \mathrm{ml}^{-1}$. Plates were incubated at $28^{\circ} \mathrm{C}$ for 3 days; then, a single colony was randomly isolated and purified. The ITS region of pureculture isolates was amplified by using ITS1F to ITS4 primers, then subjected to Sanger sequencing.

Because most of the isolated fungi were in the genus Fusarium, two Fusarium isolates (T49: Fusarium oxysporum f. sp. cumini strain F11; and T19: uncultured Fusarium clone ITS_63) were further tested to assess their pathogenicity to $R$. glutinosa seedlings under sterile conditions. In detail, tissue culture seedlings of $R$. glutinosa were incubated on Murashige-Skoog (MS) medium (supplemented with 6-BA at $0.2 \mathrm{mg} \mathrm{liter}^{-1}$, indole-3-butyric acid at $0.2 \mathrm{mg} \mathrm{liter}^{-1}$, and sucrose at $30 \mathrm{~g} \mathrm{liter}^{-1}$ ) for 45 days. Then, the mycelium of Fusarium isolates was inoculated on the MS medium. Each treatment had three replicates. The tissue culture vessels were placed in a growth chamber at $25^{\circ} \mathrm{C}$ with a photoperiod of $16 \mathrm{~h}$ of light and $8 \mathrm{~h}$ of darkness for an additional 20 days to observe the growth status and disease symptoms of $R$. glutinosa.

Effects of $F$. oxysporum metabolites on the growth of antagonistic Pseudomonas aeruginosa. A spore suspension $\left(2.4 \times 10^{6}\right.$ spores $/ \mathrm{ml}$ of sterile water) of $F$. oxysporum was inoculated into $100 \mathrm{ml}$ of potato-sugar-agar broth medium. After 8 days of incubation in a liquid shaker at $180 \mathrm{rpm}$ and $28^{\circ} \mathrm{C}$, the fermentation broth was filtered through $0.22-\mu \mathrm{m}$ filters. Then, $0,0.125$, $0.25,0.5,0.75$, and $1 \mathrm{ml}$ of culture filtrate was added into an eightfold dilution of Luria-Bertani (LB) broth medium and diluted up to $5 \mathrm{ml}$. Pseudomonas aeruginosa $(30 \mu \mathrm{l})$, previously isolated from $R$. glutinosa rhizosphere with antagonistic activity against F. oxysporum (Wu et al. 2015), was inoculated into LB broth medium. Each concentration of $F$. oxysporum culture filtrate had four replicates (namely, four tubes). After $8 \mathrm{~h}$ of incubation in a liquid shaker at $200 \mathrm{rpm}$ and $28^{\circ} \mathrm{C}$, optical density (OD) of bacterial culture was determined at $600 \mathrm{~nm}$ with a plate reader (Multiskan MK3; Thermo Scientific, Shanghai, China).

Because three mycotoxins-deoxynivalenol (DON), 3-acetyldeoxynivalenol (3A-DON), and 15-O-acetyl-4-deoxynivalenol (15ADON) - were detected in the fermentation broth of $F$. oxysporum T49 (Supplementary Fig. S2), a series of different final concentrations $(0,0.02,0.04,0.08,0.16$, and $0.32 \mu \mathrm{g} / \mathrm{ml})$ of mycotoxin mixtures consisting of equivalent amounts of these three toxins was further applied to test their inhibitory effects on the growth of $P$. aeruginosa. In detail, mycotoxin mixtures were prepared using the standard powders (Sigma-Aldrich, St. Louis), filtered through $0.22-\mu \mathrm{m}$ filters, then added into the eightfold dilution of LB broth medium. P. aeruginosa $(30 \mu \mathrm{l})$ was inoculated and stirred at $180 \mathrm{rpm}$ and $28^{\circ} \mathrm{C}$ for $8 \mathrm{~h}$; then, OD value at $600 \mathrm{~nm}$ was determined. Each treatment had four replicates.

Statistical analyses. One-way analysis of variance followed by Fisher's least significant difference test $(P<0.05)$ was applied for multiple comparisons to determine the significance of the differences of the relative abundance of the fungal taxa, $\alpha$ diversity indices, and OD of bacterial suspensions among treatments.

\section{RESULTS}

OTU cluster and species annotation. Samples obtained $1,192,139$ effective tags, with an average of 44,153 effective tags per sample (Supplementary Table S1). The N90 length was approximately 337 to $340 \mathrm{bp}$, which is close to the size of ITS2 amplicons. The Good's coverage estimates for all soil samples were $\geq 0.950$, representing good sampling depth. The number of sequences assignable to known taxa in database gradually decreased from phylum to species. In general, 80.5, 73.1, 71.8, 65.5, 51.2, and $40.3 \%$ of the effective sequences could be assigned to phylum, class, order, family, genus, and species level, respectively.

The $\boldsymbol{\alpha}$ and $\boldsymbol{\beta}$ diversity analysis. In total, 1,741, 1,666, 1,902 $1,553,1,721,1,498,1,636,1,978$, and 1,773 OTU were obtained at the level of $97 \%$ similarity from CK, RNP, RSM, RTM, RFM, BNP, BSM, BTM, and BFM, respectively. The control soil showed higher fungal community evenness and Shannon diversity than the $R$. glutinosa-planted rhizosphere soils (RNP, RSM, RTM, and RFM) (Fig. 1). No significant differences were observed for the $R$. glutinosa-planted soils in the rhizosphere. However, the results from the bulk soil showed that, with the increasing years of monoculture, both evenness and diversity indices were first decreased and then increased. Moreover, the evenness and Shannon diversity indices were significantly higher in BFM than in BNP and BSM $(P<0.05)$.

NMDS and clustering analysis based on Bray-Curtis dissimilarity showed that the fungal community in rhizosphere soils (RNP, RSM, RTM, and RFM) was obviously different from that in bulk soils (BNP, BSM, BTM, and BFM) (Fig. 2). The rhizosphere soil samples were clustered together. The fallow soil samples with no $R$. glutinosa cultivation (CK) were more similar to the bulk soil samples and were clustered together and separated from the rhizosphere soil samples. Moreover, it was found that longer-term monocultured bulk soils (BTM and BFM) were more similar to the rhizosphere soils as compared with the shorter-term monocultured bulk soils (BNP and BSM). In addition, the fungal community in both bulk and rhizosphere soils gradually changed with the increasing years of monoculture (Fig. 2).

Venn diagram analysis. Venn diagram analysis based on $\mathrm{CK}$ and rhizosphere soil samples (RNP, RSM, RTM, and RFM) showed that the cultivation of $R$. glutinosa led to the appearance of 1,968 OTU exclusively found in at least one rhizosphere sample (RNP, RSM, RTM, and RFM), which were assigned to the genera Fusarium, Mortierella, Thanatephorus, Cladosporium, Zopfiella, Rhizoctonia, and so on (Supplementary Fig. S3). Venn diagram analysis based on CK and bulk soil samples (BNP, BSM, BTM, and BFM) showed that the number of OTU exclusively found in at least one bulk sample (BNP, BSM, BTM, and BFM) was 2,179, which were mainly assigned to the genera Leucoagaricus, Eocronartium, Cyphellophora, Rhizoctonia, Zopfiella, Mortierella, Fusarium, and so on.

Venn diagram analysis of rhizosphere soil samples (RNP, RSM, RTM, and RFM) showed that the number of OTU shared in these four treatments (defined as the core rhizosphere microbiome among 
R. glutinosa-planted soils) was 583 (Supplementary Fig. S4). The number of OTU found in at least one monocultured rhizosphere soil (RSM, RTM, and RFM) and not found in RNP was 1,530. Venn diagram analysis of bulk soil samples (BNP, BSM, BTM, and BFM) showed that the number of shared OTU (defined as core bulk microbiome among $R$. glutinosa-planted soils) was 435 (Supplementary Fig. S5). The number of OTU found in at least one monocultured bulk soil (BSM, BTM, and BFM) and not found in BNP was 2,028. Taxonomic analysis showed that the shared OTU among RNP, RSM, RTM, and RFM (core rhizosphere microbiome) were mostly assigned to the genera Fusarium, Ascobolus, Zopfiella, Actinomucor, Myrothecium, Chaetomium, Podospora, Thielavia, and so on. Similarly, most of them were also identified in the core bulk microbiome. Moreover, most of the species exclusively found in the NP treatment belonged to the genera Cristinia, Eocronartium, Actinomucor, Saccharicola, and Mortierella (in RNP) and Auricularia, Ascobolus, Chaetomium, Eocronartium, Conocybe, and so on (in BNP). Some of them (i.e., Actinomucor, Saccharicola, and Mortierella) were commonly reported as antagonists against phytotoxic compounds (i.e., Actinomucor elegans) or against phytopathogenic fungi. In contrast, most of the species exclusively found in the monocultured rhizosphere soils (RSM, RTM, and RFM) belonged to the genera Thanatephorus, Septoria, Fusarium, Monosporascus, Alternaria, and Cephaliophora, which were commonly reported as plant pathogens in various crops. Several species belonging to reported pathogens Thielaviopsis, Fusarium, and Rhizoctonia were also identified in the monocultured bulk soils (BSM, BTM, and BFM).
Shifts in soil fungal community structure under consecutive monoculture. Taxonomic annotation displayed that 5, 15, $46,89,194,349$ taxa were identified in all the samples at the levels of phylum, class, order, family, genus and species, respectively. There was no obvious difference in the number of identified taxa among different treatments at each level. However, there were significant differences in the relative abundances of some specific taxa at different classification levels (Fig. 3).

Five phyla were found across all samples, with Ascomycota and Basidiomycota as the dominant phyla, representing 65.68 and $13.21 \%$ of the total classified fungal sequences, respectively (Supplementary Fig. S6). In the rhizosphere, compared with the NP (RNP), the relative abundance of Ascomycota spp. was increased whereas the number of Zygomycota spp. was reduced under consecutive monoculture. Moreover, it should be noted that RNP has the highest relative abundance of Zygomycota spp. However, the changes of these phyla in the bulk soil displayed no consistent pattern.

At the family level, the relative abundances of species of Pleosporaceae, Corticiaceae, and Magnaporthaceae were significantly $(P<0.05)$ higher in CK than in $R$. glutinosa-planted rhizosphere and bulk soils (Fig. 3). In both rhizosphere and bulk soils, the relative abundances of the families Nectriaceae, Ceratobasidiaceae, and Plectosphaerellaceae significantly $(P<$ 0.05 ) increased with the extended monoculture. Moreover, the abundances of families Ceratobasidiaceae and Plectosphaerellaceae gradually increased with the increasing years of monoculture in both rhizosphere and bulk soils. Family Chaetomiaceae also
A

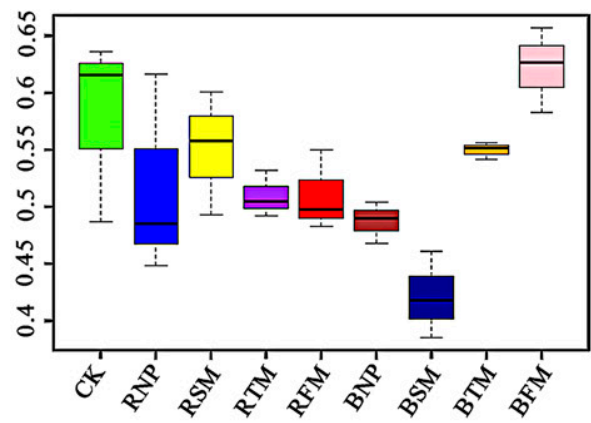

B

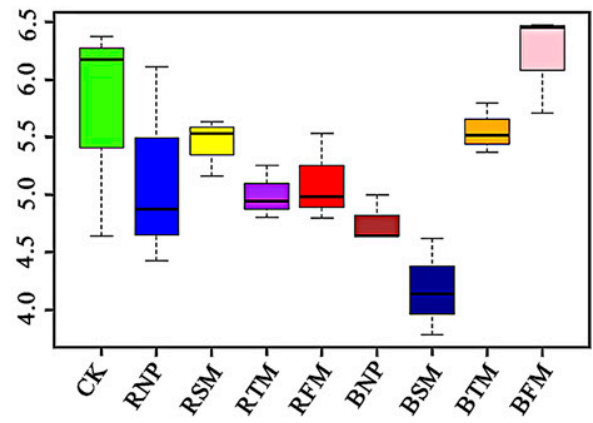

C

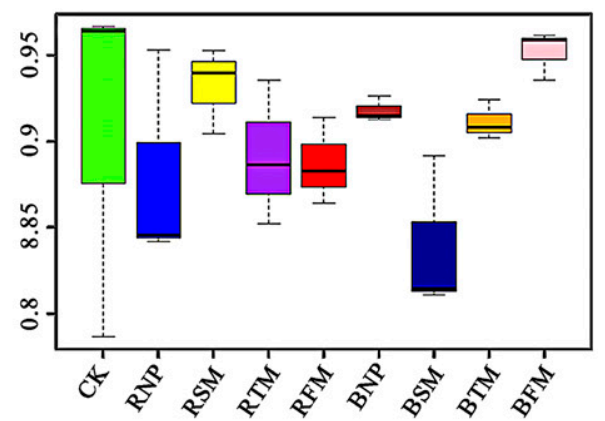

Fig. 1. Calculations of A, Pielou's evenness index and $\mathbf{B}$, Shannon and $\mathbf{C}$, Simpson diversity indices in different soil samples. CK represents the fallow soil samples with no Rehmannia glutinosa cultivation. RNP, RSM, RTM, and RFM represent the rhizosphere soil from the newly planted, 2-year, 3-year, and 4-year monocultured $R$. glutinosa plants, respectively. BNP, BSM, BTM, and BFM represent the bulk soil from the newly planted, 2-year, 3-year, and 4-year monocultured $R$. glutinosa plants, respectively.
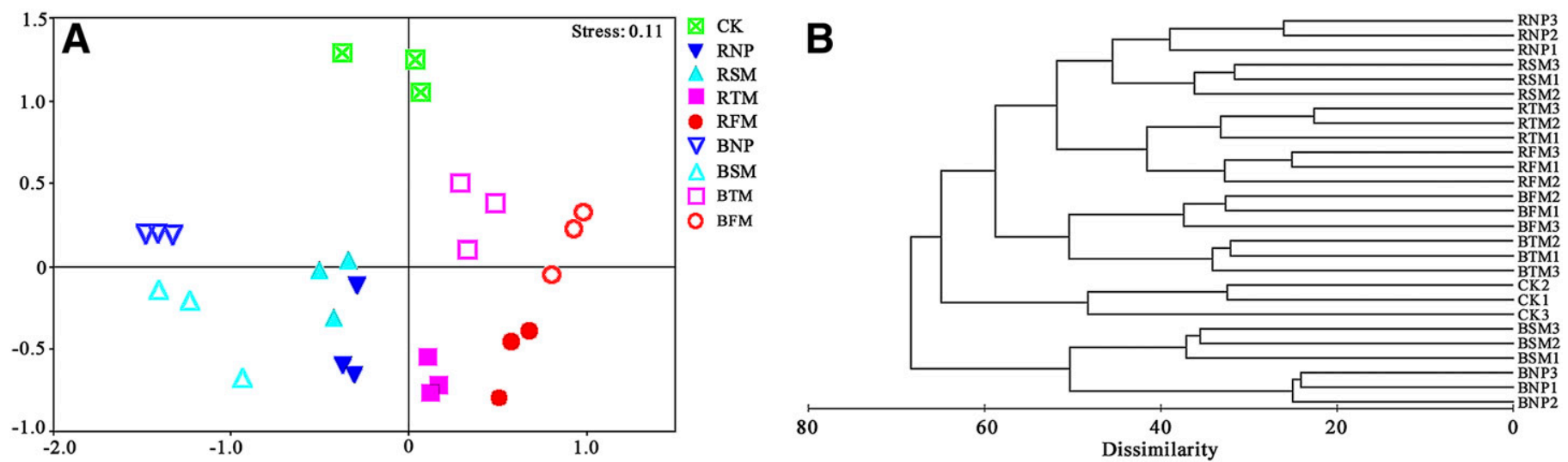

Fig. 2. A, Nonmetric multidimensional scaling ordination and $\mathbf{B}$, clustering analysis of fungal communities among different soil categories. CK represents the fallow soil samples with no Rehmannia glutinosa cultivation. RNP, RSM, RTM, and RFM represent the rhizosphere soil from the newly planted, 2-year, 3-year, and 4-year monocultured $R$. glutinosa plants, respectively. BNP, BSM, BTM, and BFM represent the bulk soil from the newly planted, 2-year, 3-year, and 4-year monocultured $R$. glutinosa plants, respectively. Numbers followed by the treatments in B represent the three replicates. 
showed a tendency to increase gradually with the extended monoculture in the rhizosphere. However, the opposite was true for the relative abundances of species of Ascobolaceae in both rhizosphere and bulk soils (Fig. 3).

Further analysis at the genus level revealed that the sum of the relative abundances of Fusarium, Cylindrocarpon, and Gibberella (belonging to the family Nectriaceae); Rhizoctonia, Thanatephorus, and Ceratobasidium (belonging to the family Ceratobasidiaceae); and Lectera and Plectosporium (belonging to the family Plectosphaerellaceae) was significantly $(P<0.05)$ higher in the longer-term CM (TM and FM) than in the NP in both rhizosphere and bulk soils (Fig. 4). For NP, SM, and TM, the sum of the abovementioned genera was significantly $(P<0.05)$ lower in bulk soils than in rhizosphere soils. However, no significant differences were observed for FM between rhizosphere and bulk soils. The sum of the abovementioned genera in BFM was even significantly $(P<$ $0.05)$ higher than that in RNP. Meanwhile, it was found that the relative abundance of Fusarium spp. was the highest among the abovementioned genera in all samples (except BFM) (Fig. 4).

Because of the high similarity of longer-term monocultured soils (TM and FM) (Fig. 2), TM and FM were combined into one group, called the longer-term CM group. Then, ANOSIM and SIMPER analysis were carried out (Supplementary Table S2). ANOSIM showed that the differences between the shorter-term and longerterm monocultured treatments in both rhizosphere and bulk soils (i.e., RNP versus consecutively monocultured rhizosphere [RCM],

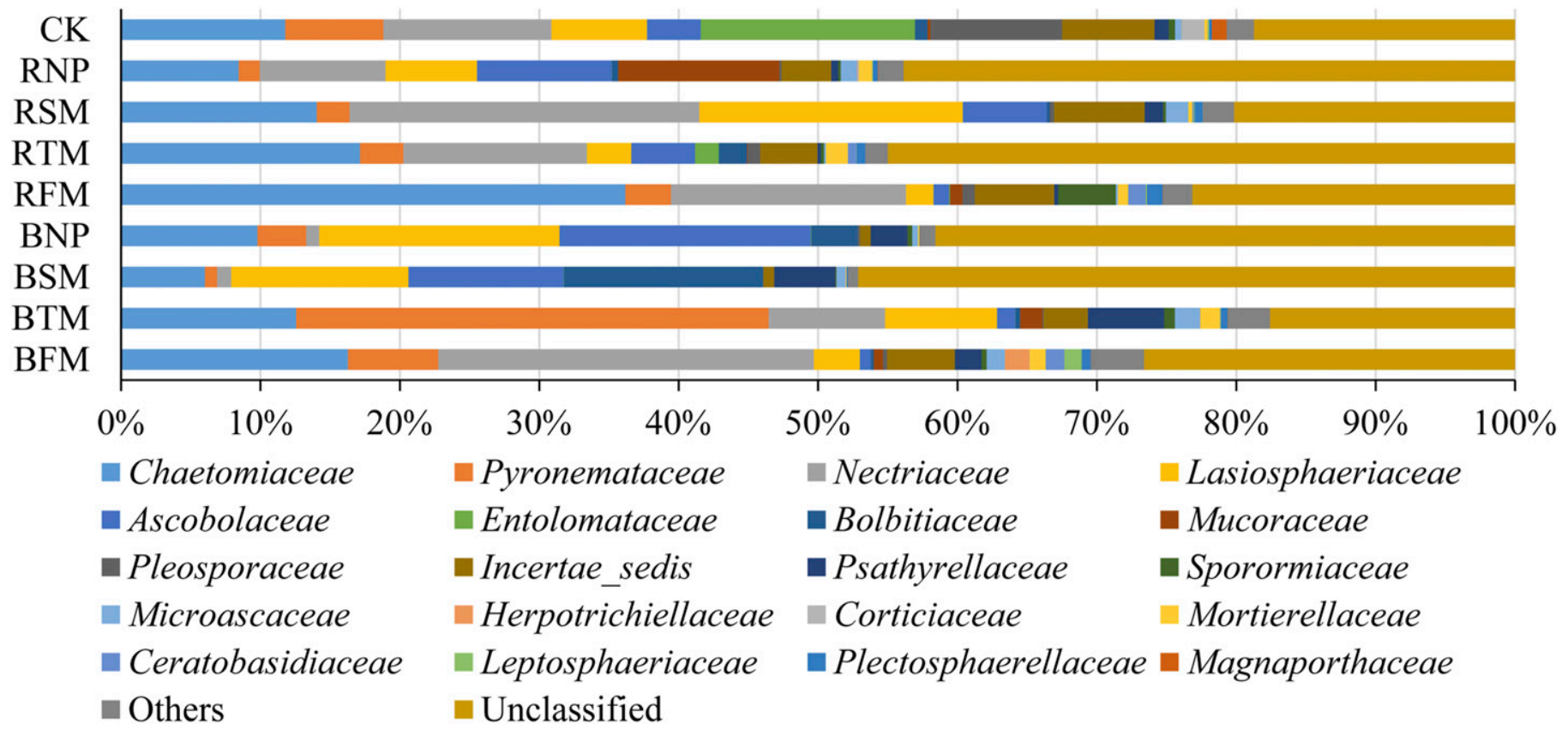

Fig. 3. Relative abundances of the top 20 fungal families in different soil categories. CK represents the fallow soil samples with no Rehmannia glutinosa cultivation. RNP, RSM, RTM, and RFM represent the rhizosphere soil from the newly planted, 2-year, 3-year, and 4-year monocultured R. glutinosa plants, respectively. BNP, BSM, BTM, and BFM represent the bulk soil from the newly planted, 2-year, 3-year, and 4-year monocultured $R$. glutinosa plants, respectively.

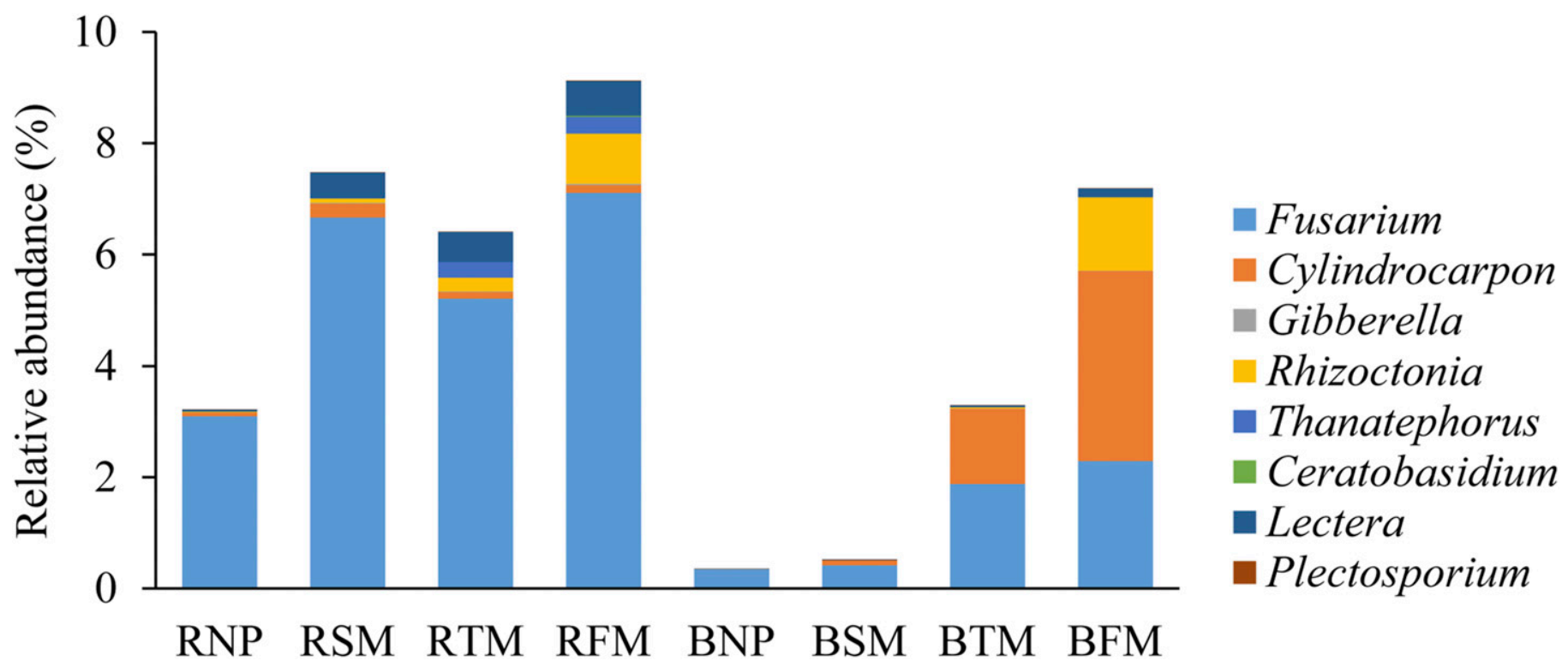

Fig. 4. Sum of relative abundances of certain genera belonging to families Nectriaceae, Ceratobasidiaceae, and Plectosphaerellaceae in different soil categories. RNP, RSM, RTM, and RFM represent the rhizosphere soil from the newly planted, 2-year, 3-year, and 4-year monocultured Rehmannia glutinosa plants, respectively. BNP, BSM, BTM, and BFM represent the bulk soil from the newly planted, 2-year, 3-year, and 4-year monocultured $R$. glutinosa plants, respectively. 
RSM versus RCM, BNP versus consecutively monocultured bulk soil $[\mathrm{BCM}]$, and $\mathrm{BSM}$ versus $\mathrm{BCM})$ were significant $(P<0.05)$. Moreover, a significant $(P<0.05)$ difference was observed between rhizosphere and bulk soils in the longer-term monocultured treatments (i.e., BCM and RCM). Further SIMPER analysis showed that the top 10 genera contributing to the dissimilarity between RNP and RCM were Actinomucor, Ascobolus, Cephaliophora, Preussia, Thielavia, Chaetomium, Fusarium, Podospora, Alternaria, and Conocybe. Among these, the relative abundances of Cephaliophora, Fusarium, and Alternaria in the rhizosphere increased with the increasing years of monoculture, whereas Actinomucor and Ascobolus showed the opposite trend. The top 10 genera contributing to the dissimilarity between BNP and BCM included Ascobolus, Podospora, Thielavia, Cylindrocarpon, Conocybe, Haematonectria, Fusarium, Pseudaleuria, Actinomucor, and Mortierella. Among these, the relative abundances of Cylindrocarpon, Haematonectria, and Fusarium in bulk soil increased with the increasing years of monoculture, whereas Ascobolus and Podospora showed the opposite trend. The top 10 genera contributing to the dissimilarity between BCM and RCM included Coprinellus, Cylindrocarpon, Fusarium, Preussia, Pseudaleuria, Haematonectria, Ascobolus, Zopfiella, Cephaliophora, and Chaetomium. Among these, the relative abundances of Fusarium and Cephaliophora were higher in rhizosphere soils than in bulk soils in the longer-term monocultured treatments (RTM versus BTM and RFM versus BFM). In addition, it was found that Fusarium was the top 10 shared genus contributing to the observed differences between RNP and $\mathrm{RCM}, \mathrm{BNP}$ and BCM, BSM and BCM, and BCM and RCM. Moreover, the relative abundance of Fusarium spp. was significantly $(P<0.05)$ higher in consecutively monocultured rhizosphere soils (RSM, RTM, and RFM) than in RNP, and was significantly $(P<0.05)$ higher in rhizosphere soils than in bulk soils (RNP versus BNP, RSM versus BSM, RTM versus BTM, and RFM versus BFM) (Fig. 4).

Isolation of potential fungal pathogens and their effects on the growth of $R$. glutinosa seedlings and antagonistic bacterium. The sequencing results of potential fungal pathogens from the rhizosphere soil of $R$. glutinosa-diseased plants are shown in Supplementary Table S3. It was found that most of the isolated fungi belonged to genus Fusarium. Some fungi (i.e., uncultured Plectosphaerella clone TVD_ITS1F-ITS4_66) belonging to the family Plectosphaerellaceae were successfully isolated in the diseased soil as well. Moreover, $F$. oxysporum and $F$. solani were isolated, both of which were successfully identified in the highthroughput sequencing results. The proportion of $F$. oxysporum was 24\%. Pathogenicity tests showed that both Fusarium clone ITS_63 (T19) and F. oxysporum f. sp. cumini strain F11 (T49) were highly pathogenic to $R$. glutinosa seedlings, especially T49. Tissue culture seedlings treated with T49 soon developed the disease symptoms, withered, and died 9 days after infection, while seedlings treated with T19 died after 18 days of inoculation.
In addition, the results in the current work found that the culture filtrate of $F$. oxysporum had a significant $(P<0.05)$ inhibitory effect on the growth of its antagonistic bacterium, $P$. aeruginosa. Furthermore, different final concentrations (from 0.04 to $0.32 \mu \mathrm{g} / \mathrm{ml}$ ) of mycotoxin mixtures, including DON, 3A-DON, and 15A-DON, could significantly $(P<0.05)$ inhibit the growth of $P$. aeruginosa (Fig. 5).

\section{DISCUSSION}

Replant disease, also known as consecutive monoculture problem, has been widely reported in various medicinal, vegetable, and horticultural plants (Dong et al. 2016; Li et al. 2014b; Li et al. 2017; Zhou and Wu 2012). Our field experiment demonstrated the severe growth inhibition effects caused by extended $R$. glutinosa monoculture. Recently, increasing studies of replant disease mainly focused on plant-soil-microbe interactions, which are critical to aboveground plant growth, health, and fitness (Berendsen et al. 2012; Huang et al. 2014; Leach et al. 2017; Wallenstein 2017; Zhang et al. 2017). Plant roots may release up to $20 \%$ of their photosynthate to the rhizosphere, which provides nutrient sources for soil microbes and shapes the rootassociated microbiome by attracting or repelling specific microbes (Lareen et al. 2016; Poole 2017). In turn, the root-associated microbiome, also referred to as the plant's second genome, could positively or negatively influence plant health and growth (Berendsen et al. 2012). Plant growth-promoting rhizobacteria and plant growthpromoting fungi have been shown to positively impact plant performance via different mechanisms (Goswami et al. 2016; Hossain et al. 2017; Naznin et al. 2014). By contrast, soilborne pathogens can exert negative effects on plant health. They compete with members of root microbiome for available nutrients and microsites and establish a parasitic relationship with the host (Chapelle et al. 2016).

It has been debated how consecutive monoculture of medicinal plants influences the composition and structure of soil fungal communities, which are crucial for maintaining soil health. In the current work, deep pyrosequencing of ITS2 rDNA amplicons was applied to assess the shifts of the rhizosphere and bulk soil fungal communities under 4-year monoculture of $R$. glutinosa. NMDS and clustering analysis revealed distinctive differences between the shorter-term (NP and SM) and longer-term (TM and FM) monocultured treatments in both rhizosphere and bulk soils. Moreover, the results revealed distinctive differences between rhizosphere and bulk soil fungal communities. It has become increasingly evident that the rhizosphere and bulk soil host contrasting microbial and faunal communities as a result of the modulation of root exudates and rhizodeposits (de Vries and Wallenstein 2017; Mendes et al. 2014). Further studies also indicated that the composition and network of the bulk soil community was relatively stable under changing environmental conditions because bulk soil harbors a diverse network of organisms with weak interactions, and the majority of
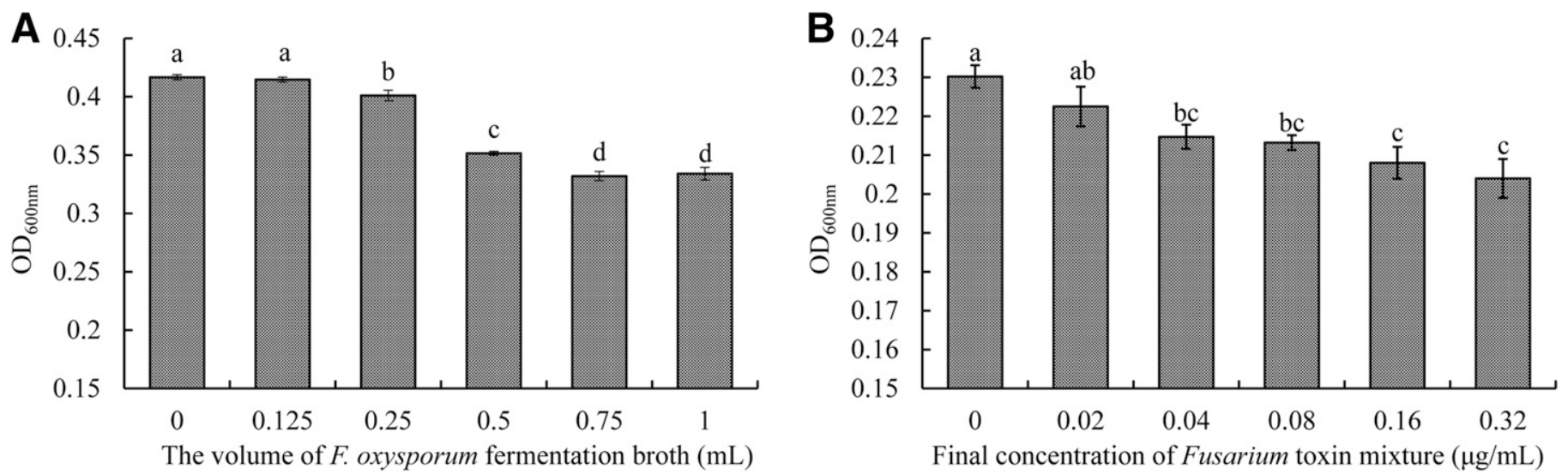

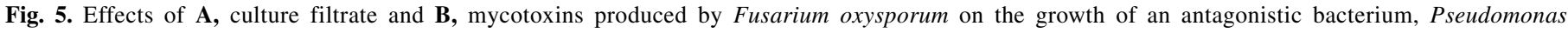
aeruginosa. 
these organisms are dormant or inactive (de Vries and Wallenstein 2017; Shi et al. 2016). In addition, it was found that consecutive monoculture of $R$. glutinosa had a greater effect on the fungal communities in rhizosphere soils than in bulk soils because the control soil without $R$. glutinosa cultivation (CK) was more similar to the fungal communities in bulk soils. However, it should be noted that soil fungal communities from longer-term monocultured bulk soils (BTM and BFM) were more similar to the rhizosphere fungal communities as compared with the shorter-term monocultured bulk soils (BNP and BSM). Similar trends were also observed in the relative abundances of certain fungal taxa such as the family Nectriaceae, genus Fusarium, suggesting that the effects of consecutive monoculture on bulk soil were significant but delayed as compared with rhizosphere soil. From the abovementioned results, we speculated that effects of consecutive monoculture practice on the fungal communities in bulk soil were relatively less pronounced but long-term consecutive monoculture (3 years or more) affected bulk soil fungal communities in ways similar to the rhizosphere communities. In turn, the composition in bulk soil, also known as seed bank, would affect the rhizosphere recruitment and networks of subsequent replanted $R$. glutinosa plants.

Soil microbes live in close association with plants and the crosstalk between plants and microbes is considered to be the key factor for soil health and plant performance (Andreote and de Cássia Pereira e Silva 2017; Berendsen et al. 2012). The results in this study showed that, compared with the control soil (CK), R. glutinosa monoculture led to the appearance of some exclusive OTU in the rhizosphere (i.e., genera Fusarium, Thanatephorus, and Rhizoctonia) and bulk soils (i.e., genera Fusarium and Rhizoctonia). Additionally, it was found that consecutive monoculture of this plant led to a significant increase in the relative abundances of the families Nectriaceae, Ceratobasidiaceae, and Plectosphaerellaceae in both rhizosphere and bulk soils. Similar trends were also observed in the sum of the abundances of Fusarium, Cylindrocarpon, and Gibberella (belonging to the family Nectriaceae); Rhizoctonia, Thanatephorus, and Ceratobasidium (belonging to the family Ceratobasidiaceae); and Lectera and Plectosporium (belonging to the family Plectosphaerellaceae). Numerous fungi of the families Nectriaceae, Ceratobasidiaceae, and Plectosphaerellaceae are important agricultural pathogens that cause root rots, wilts, and damping-off in many crops around the world (Ajayi-Oyetunde and Bradley 2018; Cannon et al. 2012; Shen et al. 2018). The current study resonates with previous findings that replant disease is attributable to an increase in soilborne pathogens along with a decrease in the beneficial microorganisms (Jiang et al. 2017; Li et al. 2014b; Nicola et al. 2017; Wu et al. 2015). Fusarium, Cylindrocarpon, and Rhizoctonia spp. are considered to be essential in the etiology of apple replant disease (Franke-Whittle et al. 2015; Mazzola et al. 2002; van Schoor et al. 2009). Similar examples could also be found in many other Chinese medicinal plants such as Panax notoginseng, P. ginseng, and Pseudostellaria heterophylla (Dong et al. 2016, 2017; Mao et al. 2014; L. K. Wu et al. 2016).

SIMPER analysis indicated that Fusarium was the top 10 shared genus that contributed to the observed differences between the NP and longer-term monocultured treatments (RNP versus RCM and $\mathrm{BNP}$ versus $\mathrm{BCM}$ ), and between the rhizosphere and bulk soils of longer-term monocultured treatment (BCM versus RCM). Similarly, the abundance of Fusarium was reported to be increased with the extended monoculture in various crops (Li et al. 2014b; L. K. Wu et al. 2016; Zhou and $\mathrm{Wu}$ 2012). Many pathogenic species of Fusarium can cause vascular diseases and crop yield losses (Fravel et al. 2003; Qiu et al. 2012). The isolated Fusarium spp. (T19 and T49) in this study were also found to be highly pathogenic to $R$. glutinosa seedlings. In addition, the culture filtrate of isolated F. oxysporum significantly repressed the growth of the antagonistic bacterium $P$. aeruginosa. Similarly, Liu et al. (2017) found that culture filtrates of Fusarium sp. ACCC36194 were strongly inhibitory to the growth of Arthrobacter isolates, which produced high levels of indole acetic acid. It has been proved that phenolic acids in the root exudates of $R$. glutinosa could promote the mycelial growth and DON toxin production of pathogenic F. oxysporum $(\mathrm{Li}$ et al. 2016; Wu et al. 2015). Mycotoxins, including fusaric acid and DON toxins, excreted by Fusarium spp. were reported to be toxic to surrounding microorganisms (Notz et al. 2002; H. M. Wu et al. 2016). This might be another reason for the decline in the beneficial Pseudomonas spp. under R. glutinosa monoculture observed in our previous study, in addition to the modulation by root exudates ( $\mathrm{Wu}$ et al. 2015). Similar to plant-microbe interactions, microbe-microbe interactions have important consequences for soil microbial community and host health (Lareen et al. 2016).

In conclusion, consecutive monoculture of $R$. glutinosa influenced the fungal community composition and abundance in both rhizosphere and bulk soils by increasing the relative abundances of fungal pathogens (i.e., Fusarium and Rhizoctonia spp.) but bulk effects developed more slowly over time in comparison with rhizosphere effects. Moreover, explosive growth and proliferation of F. oxysporum and their toxic secondary metabolites (i.e., mycotoxins) could inhibit the growth of the beneficial Pseudomonas sp., which aggravated the soil microbial dysbiosis under $R$. glutinosa consecutive monoculture and, thus, led to the replant disease. This study gave an insight into how consecutive monoculture shapes the soil fungal community and provided a clear indication for screening pathogens and corresponding antagonistic microbes of $R$. glutinosa. Further works using pyrosequencing with higher sequencing depth or other techniques (i.e., metagenomics sequencing) are needed to study the shifts in the soil microbial community in the $R$. glutinosa monoculture regime. To restore soil health and overcome the replant disease of $R$. glutinosa, further studies will also be performed to manipulate the rhizosphere microbiome or manipulate plant traits that are related to interactions with microbes.

\section{LITERATURE CITED}

Ajayi-Oyetunde, O. O., and Bradley, C. A. 2018. Rhizoctonia solani: Taxonomy, population biology, and management of rhizoctonia seedling disease of soybean. Plant Pathol. 67:3-17.

Andreote, F. D., and de Cássia Pereira e Silva, M. 2017. Microbial communities associated with plants: Learning from nature to apply it in agriculture. Curr. Opin. Microbiol. 37:29-34.

Benizri, E., Piutti, S., Verger, S., Pagès, L., Vercambre, G., Poessel, J. L., and Michelot, P. 2005. Replant diseases: Bacterial community structure and diversity in peach rhizosphere as determined by metabolic and genetic fingerprinting. Soil Biol. Biochem. 37:1738-1746.

Berendsen, R. L., Pieterse, C. M. J., and Bakker, P. A. H. M. 2012. The rhizosphere microbiome and plant health. Trends Plant Sci. 17:478-486.

Cannon, P., Buddie, A., Bridge, P., de Neergaard, E., Lübeck, M., and Askar, M. 2012. Lectera, a new genus of the Plectosphaerellaceae for the legume pathogen Volutella colletotrichoides. MycoKeys 3:23-36.

Chapelle, E., Mendes, R., Bakker, P. A. H., and Raaijmakers, J. M. 2016. Fungal invasion of the rhizosphere microbiome. ISME J. 10:265-268.

Chen, T., Lin, S., Wu, L. K., Lin, W. X., and Sampietro, D. A. 2015. Soil sickness: Current status and future perspectives. Allelopathy J. 36:167-195.

Deshpande, V., Wang, Q., Greenfield, P., Charleston, M., Porras-Alfaro, A., Kuske, C. R., Cole, J. R., Midgley, D. J., and Tran-Dinh, N. 2016. Fungal identification using a Bayesian classifier and the Warcup training set of internal transcribed spacer sequences. Mycologia 108:1-5.

de Vries, F. T., and Wallenstein, M. D. 2017. Below-ground connections underlying above-ground food production: A framework for optimising ecological connections in the rhizosphere. J. Ecol. 105:913-920.

Dong, L., Xu, J., Feng, G., Li, X., and Chen, S. 2016. Soil bacterial and fungal community dynamics in relation to Panax notoginseng death rate in a continuous cropping system. Sci. Rep. 6: Article 31802.

Dong, L., Xu, J., Zhang, L., Wei, G., Su, H., Yang, J., Qian, J., Xu, R., Liao, B., Shen, L., Wu, M., Cheng, R., and Wu, M. 2017. Inoculation of biocontrol bacteria alleviated Panax ginseng replanting problem. Online publication. bioRxiv

Franke-Whittle, I. H., Manici, L. M., Insam, H., and Stres, B. 2015. Rhizosphere bacteria and fungi associated with plant growth in soils of three replanted apple orchards. Plant Soil 395:317-333.

Fravel, D., Olivain, C., and Alabouvette, C. 2003. Fusarium oxysporum and its biocontrol. New Phytol. 157:493-502. 
Goswami, D., Thakker, J. N., and Dhandhukia, P. C. 2016. Portraying mechanics of plant growth promoting rhizobacteria (PGPR): A review. Online publication. Cogent Food Agric. 2.

Haichar, F. Z., Marol, C., Berge, O., Rangel-Castro, J. I., Prosser, J. I., Balesdent, J., Heulin, T., and Achouak, W. 2008. Plant host habitat and root exudates shape soil bacterial community structure. ISME J. 2:1221-1230.

Han, J., Pang, X., Liao, B., Yao, H., Song, J., and Chen, S. 2016. An authenticity survey of herbal medicines from markets in China using DNA barcoding. Sci. Rep. 6: Article 18723.

Hewavitharana, S. S., and Mazzola, M. 2016. Carbon source-dependent effects of anaerobic soil disinfestation on soil microbiome and suppression of Rhizoctonia solani AG-5 and Pratylenchus penetrans. Phytopathology 106: 1015-1028.

Hossain, M. M., Sultana, F., and Islam, S. 2017. Plant growth-promoting fungi (PGPF): Phytostimulation and induced systemic resistance. Pages 135-191 in: Plant-Microbe Interactions in Agro-Ecological Perspectives. D. P. Singh, H. B. Singh, and R. Prabha, eds. Springer, Singapore.

Huang, L. F., Song, L. X., Xia, X. J., Mao, W. H., Shi, K., Zhou, Y. H., and Yu, J. Q. 2013. Plant-soil feedbacks and soil sickness: From mechanisms to application in agriculture. Chem. Ecol. 39:232-242.

Huang, X. F., Chaparro, J. M., Reardon, K. F., Zhang, R., Shen, Q., and Vivanco, J. M. 2014. Rhizosphere interactions: Root exudates, microbes, and microbial communities. Botany 92:267-275.

Jiang, J., Song, Z., Yang, X., Mao, Z., Nie, X., Guo, H., and Peng, X. 2017. Microbial community analysis of apple rhizosphere around Bohai Gulf. Sci. Rep. 7: Article 8918.

Lakshmanan, V., Selvaraj, G., and Bais, H. P. 2014. Functional soil microbiome: Belowground solutions to an aboveground problem. Plant Physiol. 166:689-700.

Lareen, A., Burton, F., and Schäfer, P. 2016. Plant root-microbe communication in shaping root microbiomes. Plant Mol. Biol. 90:575-587.

Leach, J. E., Triplett, L. R., Argueso, C. T., and Trivedi, P. 2017. Communication in the Phytobiome. Cell 169:587-596.

Leinfelder, M. M., and Merwin, I. A. 2006. Rootstock selection, preplant soil treatments, and tree planting positions as factors in managing apple replant disease. HortScience 41:394-401.

Li, M., Yang, Y., Feng, F., Zhang, B., Chen, S., Yang, C., Gu, L., Wang, F., Zhang, J., Chen, A., Lin, W., Chen, X., and Lin, W. 2017. Differential proteomic analysis of replanted Rehmannia glutinosa roots by iTRAQ reveals molecular mechanisms for formation of replant disease. BMC Plant Biol. 17:116.

Li, X. G., Ding, C. F., Hua, K., Zhang, T. L., Zhang, Y. N., Zhao, L., Yang, Y. R., Liu, J. G., and Wang, X. X. 2014a. Soil sickness of peanuts is attributable to modifications in soil microbes induced by peanut root exudates rather than to direct allelopathy. Soil Biol. Biochem. 78:149-159.

Li, X. G., Ding, C. F., Zhang, T. L., and Wang, X. X. 2014b. Fungal pathogen accumulation at the expense of plant-beneficial fungi as a consequence of consecutive peanut monoculturing. Soil Biol. Biochem. 72:11-18.

Li, Z. F., He, C. L., Wang, Y., Li, M. J., Dai, Y. J., Wang, T., and Lin, W. X. 2016. Enhancement of trichothecene mycotoxins of Fusarium oxysporum by ferulic acid aggravates oxidative damage in Rehmannia glutinosa Libosch. Sci. Rep. 6: Article 33962.

Liu, J., Wang, X., Zhang, T., and Li, X. 2017. Assessment of active bacteria metabolizing phenolic acids in the peanut (Arachis hypogaea L.) rhizosphere. Microbiol. Res. 205:118-124.

Manici, L. M., and Caputo, F. 2009. Fungal community diversity and soil health in intensive potato cropping systems of the east Po valley, northern Italy. Ann. Appl. Biol. 155:245-258.

Mao, Z. S., Long, Y. J., Zhu, Y. Y., Zhu, S. S., He, X. H., and Chen, Z. J. 2014. First report of Cylindrocarpon destructans var. destructans causing black root rot of sanqi (Panax notoginseng) in China. Plant Dis. 98:162.

Mazzola, M., Granatstein, D. M., Elfving, D. C., Mullinix, K., and Gu, Y. H. 2002. Cultural management of microbial community structure to enhance growth of apple in replant soils. Phytopathology 92:1363-1366.

Mendes, L. W., Kuramae, E. E., Navarrete, A. A., Van Veen, J. A., and Tsai, S. M. 2014. Taxonomical and functional microbial community selection in soybean rhizosphere. ISME J. 8:1577-1587.

Miethling, R., Wieland, G., Backhaus, H., and Tebbe, C. C. 2000. Variation of microbial rhizosphere communities in response to crop species, soil origin, and inoculation with Sinorhizobium meliloti L33. Microb. Ecol. 40:43-56.

Naznin, H. A., Kiyohara, D., Kimura, M., Miyazawa, M., Shimizu, M., and Hyakumachi, M. 2014. Systemic resistance induced by volatile organic compounds emitted by plant growth-promoting fungi in Arabidopsis thaliana. PLoS One 9:e86882.

Nicola, L., Turco, E., Albanese, D., Donati, C., Thalheimer, M., Pindo, M., Insam, H., Cavalieri, D., and Pertot, I. 2017. Fumigation with dazomet modifies soil microbiota in apple orchards affected by replant disease. Appl. Soil Ecol. 113:71-79.

Notz, R., Maurhofer, M., Dubach, H., Haas, D., and Défago, G. 2002. Fusaric acidproducing strains of Fusarium oxysporum alter 2, 4-diacetylphloroglucinol biosynthetic gene expression in Pseudomonas fluorescens CHA0 in vitro and in the rhizosphere of wheat. Appl. Environ. Microbiol. 68:2229-2235.

Park, S. U., Park, N. I., Kim, Y. K., Suh, S. Y., Eom, S. H., and Lee, S. Y. 2009. Application of plant biotechnology in the medicinal plant, Rehmannia glutinosa Liboschitz. J. Med. Plants Res. 3:1258-1263.

Poole, P. 2017. Shining a light on the dark world of plant root-microbe interactions. Proc. Natl. Acad. Sci. USA 114:4281-4283.

Qiu, M., Zhang, R., Xue, C., Zhang, S., Li, S., Zhang, N., and Shen, Q. 2012. Application of bio-organic fertilizer can control Fusarium wilt of cucumber plants by regulating microbial community of rhizosphere soil. Biol. Fertil. Soils 48:807-816

Shen, Z., Penton, C. R., Lv, N., Xue, C., Yuan, X., Ruan, Y., Li, R., and Shen, Q. 2018. Banana Fusarium wilt disease incidence is influenced by shifts of soil microbial communities under different monoculture spans. Microb. Ecol. 75:739-750

Shi, S., Nuccio, E. E., Shi, Z. J., He, Z., Zhou, J., and Firestone, M. K. 2016. The interconnected rhizosphere: High network complexity dominates rhizosphere assemblages. Ecol. Lett. 19:926-936.

Tan, Y., Cui, Y., Li, H., Kuang, A., Li, X., Wei, Y., and Ji, X. 2017. Diversity and composition of rhizospheric soil and root endogenous bacteria in Panax notoginseng during continuous cropping practices. J. Basic Microbiol. 57: 337-344.

Toju, H., Tanabe, A. S., Yamamoto, S., and Sato, H. 2012. High-coverage ITS primers for the DNA-based identification of ascomycetes and basidiomycetes in environmental samples. PLoS One 7:e40863.

van Schoor, L., Denman, S., and Cook, N. C. 2009. Characterisation of apple replant disease under South African conditions and potential biological management strategies. Sci. Hortic. (Amsterdam) 119:153-162.

Wallenstein, M. D. 2017. Managing and manipulating the rhizosphere microbiome for plant health: A systems approach. Rhizosphere 3:230-232.

Wardle, D. A., Bardgett, R. D., Klironomos, J. N., Setälä, H., Van Der Putten, W. H., and Wall, D. H. 2004. Ecological linkages between aboveground and belowground biota. Science 304:1629-1633.

Wu, H. M., Wu, L. K., Wang, J. Y., Zhu, Q., Lin, S., Xu, J. H., Zheng, C. L., Chen, J., Qin, X. J., Fang, C. X., Zhang, Z. X., Azeem, S., and Lin, W. X. 2016. Mixed phenolic acids mediated proliferation of pathogens Talaromyces helicus and Kosakonia sacchari in continuously monocultured Radix pseudostellariae rhizosphere soil. Front. Microbiol. 7:335.

Wu, L. K., Chen, J., Wu, H. M., Wang, J. Y., Wu, Y. H., Lin, S., Khan, M. U., Zhang, Z., and Lin, W. X. 2016. Effects of consecutive monoculture of Pseudostellaria heterophylla on soil fungal community as determined by pyrosequencing. Sci. Rep. 6: Article 26601.

Wu, L. K., Chen, J., Xiao, Z. X., Zhu, X. C., Wang, J. Y., Wu, H. M., Wu, Y. H., Zhang, Z. Y., and Lin, W. X. 2018. Barcoded pyrosequencing reveals a shift in the bacterial community in the rhizosphere and rhizoplane of Rehmannia glutinosa under consecutive monoculture. Int. J. Mol. Sci. 19: 850.

Wu, L. K., Li, Z. F., Li, J., Khan, M. A., Huang, W. M., Zhang, Z. Y., and Lin, W. X. 2013. Assessment of shifts in microbial community structure and catabolic diversity in response to Rehmannia glutinosa monoculture. Appl. Soil Ecol. 67:1-9.

Wu, L. K., Wang, J. Y., Huang, W. M., Wu, H. M., Chen, J., Yang, Y. Q., Zhang, Z. Y., and Lin, W. X. 2015. Plant-microbe rhizosphere interactions mediated by Rehmannia glutinosa root exudates under consecutive monoculture. Sci. Rep. 5: Article 15871

Xiong, W., Li, R., Ren, Y., Liu, C., Zhao, Q., Wu, H., Jousset, A., and Shen, Q. 2017. Distinct roles for soil fungal and bacterial communities associated with the suppression of vanilla Fusarium wilt disease. Soil Biol. Biochem. 107:198-207.

Xu, L., Ravnskov, S., Larsen, J., Nilsson, R. H., and Nicolaisen, M. 2012. Soil fungal community structure along a soil health gradient in pea fields examined using deep amplicon sequencing. Soil Biol. Biochem. 46:26-32.

Yang, Q., Wang, R., Xu, Y., Kang, C., Miao, Y., and Li, M. 2016. Dynamic change of the rhizosphere microbial community in response to growth stages of consecutively monocultured Rehmanniae glutinosa. Biologia 71: 1320-1329.

Yang, Y. H., Chen, X. J., Chen, J. Y., Xu, H. X., Li, J., and Zhang, Z. Y. 2011. Differential miRNA expression in Rehmannia glutinosa plants subjected to continuous cropping. BMC Plant Biol. 11:53.

Yang, Y. H., Li, M. J., Chen, X. J., Wang, P. F., Wang, F. Q., Lin, W. X., Yi, Y. J., Zhang, Z. W., and Zhang, Z. Y. 2014. De novo characterization of the Rehmannia glutinosa leaf transcriptome and analysis of gene expression associated with replanting disease. Mol. Breed. 34:905-915.

Zhang, R., Vivanco, J. M., and Shen, Q. 2017. The unseen rhizosphere root-soil-microbe interactions for crop production. Curr. Opin. Microbiol. $37: 8-14$.

Zhou, X., and Wu, F. 2012. p-Coumaric acid influenced cucumber rhizosphere soil microbial communities and the growth of Fusarium oxysporum f. sp. cucumerinum Owen. PLoS One 7:e48288. 